C2020. Licensed under the Creative Commons Attribution-NonCommercial-NoDerivatives 4.0 International http://creativecommons.org/about/downloads

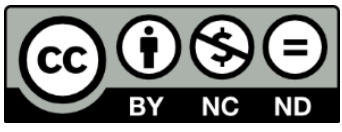

This is not the version of record. The published version can be found at https://doi.org/10.1016/j.matpr.2020.06.075 


\title{
Experimental Investigation on Energy Absorption of Auxetic Structures
}

\author{
Milad Najafi ${ }^{\mathrm{a}}$, Hamed Ahmadi ${ }^{\mathrm{a}}$, Gholamhossein Liaghat ${ }^{\mathrm{a}, \mathrm{b}}$ \\ ${ }^{a}$ Faculty of Mechanical Engineering, Tarbiat Modares University, Tehran, Iran \\ ${ }^{b}$ School of Mechanical \& Aerospace Engineering, Kingston University, London, United Kingdom
}

\begin{abstract}
The human being has always been looking for optimal use of his surrounding materials and over the years, has managed to invent various structures with special properties. Lattice structures are widely used in various applications due to their lower weight and desirable compressive strength. An example of these structures is the honeycomb that is very popular and many studies have been done about it. A new type of lattice structures is auxetic structure that has negative Poisson's ratio due to its geometry. This characteristic has caused auxetic structures to have unique properties such as high shear strength, indentation resistance and energy absorption. Investigation of energy absorption of auxetic structures is a subject that has not been studied in researches. In this study, the ability of some auxetic structure for absorbing energy is investigated at quasi-static and low velocity impact transverse loading. Specimens with three types of geometries (re-entrant, arrowhead and anti-tetra chiral) are fabricated using additive manufacturing method (3D printing). Discussion about energy absorption and failure mechanisms of all three structures were carried out and compared in both types of loading.
\end{abstract}

Keywords: Auxetic Structures, Experimental Analysis, Energy Absorption;

\section{Introduction}

Materials and structures that are used as energy absorbers, should have low weight and high compressive stiffness in addition to high energy absorption [1] and the latticed structures are among the suitable choices that can be used for this purpose [2]. Inspired by the latticed structures that have been found in nature, humans have succeeded in designing and constructing latticed structures with various geometries as energy absorbers [3]. One of the most wellknown and most used structures is the honeycomb structure, with a lot of research and studies done about it. Most of the materials that exist in nature have a positive Poisson's ratio. There are new types of structures and materials that have a negative Poisson's ratio. These materials and structures are called auxetic metamaterials [4]. The term "metamaterials" was initially used within the context of optics and electromagnetism, but today refers to all materials engineered to exhibit novel properties not usually found in nature. The important feature of auxetic metamaterials, which is negative Poisson's ratio, has given these metamaterials unique properties. In fact, these materials and structures behave unlike conventional materials under uniaxial loading. As shown in fig.1, unlike conventional materials and structures that undergo contraction and collapse under uniaxial loading, the auxetic materials and structures undergo expansion under uniaxial tensile loading. As well as in uniaxial compressive loading, auxetic materials and structures contract and shrink, while conventional materials expand under the same conditions [5]. This characteristic, gives unique properties such as variable permeability, shear strength, high tensile strength, high stiffness, high compressive strength and high energy absorption to these materials and structures [5].

\footnotetext{
* Corresponding author. Tel.: +98-21-82883391

E-mail address: h_ahmadi@modares.ac.ir
} 
Fig. 1. Deformation modes in tensile and compressive loading (dashed line figures indicate the material before deformation): a) non-auxetic material b) auxetic material.

In 1982 Gibson et al. [6] presented the first two-dimensional cellular structure with a negative Poisson's ratio in the form of re-entrant. The introduction of the first auxetic material dated back to 1987, when Lakes [7] reported the behavior of the first foam structures with negative Poisson's ratio. Four years later, materials that have negative Poisson's ratio were named by Evans et al. [8] as auxetic materials. Rafsanjani and Pasini [9], inspired by the ancient geometric motifs in the ancient Kharraghan twin tomb towers in Iran, presented a new kind of auxetic structures. Auxetic structures, due to their construction geometry, have a negative Poisson's ratio and are used to build them from conventional materials with positive Poisson's ratio. The most well-known auxetic structure is re-entrant structure and many studies have been done about it. Yang et al. [10] investigated the energy absorption of the reentrant auxetic structure and compared it with the honeycomb structure, which is a non-auxetic structure and found that the re-entrant structure shows a better performance in the energy absorption with the aim of using them in body protective pads. By performing a structural modification in the geometry of re-entrant structure, Ingrole et al. [11] compared the mechanical properties of non-auxetic honeycomb structure and re-entrant auxetic structure under quasi-static compression and found that despite the higher energy absorption of the re-entrant structure than the honeycomb structure, the specific energy absorption of the honeycomb structure is higher. Moreover, by performing a structural modification in the geometry of re-entrant structure, the energy absorption and specific energy absorption of this structure increased by $30 \%$ and $13 \%$, respectively. Imbalzano et al. [12] by numerical and experimental comparison of the resistance of the honeycomb structure and the re-entrant auxetic structure to the explosion, found that the re-entrant structure, due to its auxetic behavior, exhibits a localized stiffness and has better resistance against the explosion. Chang et al. [13] also with experimentally and numerically investigating the reentrant structure and the honeycomb, found that the performance of the re-entrant auxetic structure in absorbing the energy of impact and close in blast loading is better than the honeycomb structure. Safikhani and Etemadi [14] presented a new model of re-entrant auxetic structure in the form of warp and woof. Hamzehei et al. [15] presented a new octagonal model that exhibited auxetic behavior in large compressive deformations. Because of the challenges and difficulties that many auxetic structures have with different geometries, an additive manufacturing method (3D printing) is appropriate method for producing these structures [4]. In this study, we investigate the energy absorption and performance of three auxetic structures with re-entrant, arrowhead and anti-tetra chiral geometries in quasi-static and low velocity impact loading.

\section{Fabrication}

\subsection{Geometry of structures}

In the present work, three different geometric auxetic structures have been investigated. Figures 2(a) to 2(c), represent the unit cells of re-entrant, arrowhead and square node anti-tetra chiral (which is called as 'anti-tetra chiral' for simplicity in this paper) structures, respectively. Design parameters for each unit cell are shown in table 1 and figure 2. The re-entrant auxetic has been studied and investigated by many researchers in recent years, and so it is the most well-known auxetic structure, and has been used as benchmark for comparing two other structures. The number of unit cells and total dimensions of structures in length, width, and height were approximately identical to make a fair comparison.

Fig. 2. Unit cell profile of each model: (a) re-entrant, (b) arrowhead, (c) anti-tetra chiral.

Table 1. Design parameters of each model.

\begin{tabular}{llllll}
\hline Structure & $\mathrm{h}(\mathrm{mm})$ & $\mathrm{l}(\mathrm{mm})$ & $\mathrm{a}(\mathrm{mm})$ & $\mathrm{t}(\mathrm{mm})$ & $\alpha\left(^{\circ}\right)$ \\
\hline Re-entrant & 10 & 5 & - & 1 & -30 \\
Arrowhead & 13.50 & 8.40 & - & 1 & 30 \\
Anti-tetra chiral & 9.80 & 9.80 & 2 & 1 & - \\
\hline
\end{tabular}




\subsection{The process of fabricating}

Three different models were fabricated using the dimensions mentioned in Table 1. Figure 3 shows the models made by the additive manufacturing process (3D printing), one of the most advanced and relatively new methods for constructing specimens. The 3Dprinter was Quantom-2025 and the base material was ABS (acrylonitrile butadiene styrene), one of the most commonly used polymeric materials in the 3D printing process. In order to find the properties of the material, tensile test specimens were made and tested according to ASTM D638 standard, and its properties are mentioned in table 2 .

Table 2. Material properties.

\begin{tabular}{lllll}
\hline Base material & $\begin{array}{l}\text { Young's } \\
\text { modulus(MPa) }\end{array}$ & Yield stress(MPa) & Poisson's ratio & Density $\left(\mathrm{g} / \mathrm{c}^{3} \mathrm{~m}^{3}\right)$ \\
\hline $\mathrm{ABS}$ & 1500 & 36 & 0.35 & 1.00 \\
\hline
\end{tabular}

To ensure that loads are applied uniformly to structures, for all structures at the top and bottom, two face sheets were designed with $1 \mathrm{~mm}$ thickness, so that loads are applied uniformly and structural deformation is done correctly. Fig. 3 shows the complete shapes of structures.

Fig. 3. Fabricated models: (a) re-entrant, (b) arrowhead, (c) anti-tetra chiral.

\section{Experimental tests}

\subsection{Quasi-static compression test}

Uniaxial compression tests of the specimens was performed using the WDW-300E Universal testing Machine at the crosshead speed of $2 \mathrm{~mm} / \mathrm{min}$. The specimens were compressed to about $50 \%$ of their initial height. Deformed shapes of specimens are shown in fig.4. The output of testing machine is a force-displacement diagram that is presented for analysis in the following sections.

Fig. 4. Deformed shapes of structures after quasi-static test: (a) re-entrant structure, (b) arrowhead structure, (c) anti-tetra chiral structure.

Fig. 5. Drop weight impact tester.

\subsection{Low velocity impact test}

The apparatus used to perform the low velocity impact tests is shown in figure 5. As shown in figure 5, drop hammer used a cylindrical platen with a $200 \mathrm{~mm}$ diameter and the total weight of striker was $9.1 \mathrm{~kg}$. The drop hammer was equipped with an accelerometer to measure the acceleration. Thus the output of tests is accelerationtime diagrams. The force values was obtained from multiplying acceleration values by the mass of striker. In these tests, the height of the striker was set at $130 \mathrm{~cm}$ which led to a theoretical $115.9 \mathrm{~J}$ amount of energy, without including the amounts of energy loss due to friction. Deformed shapes of specimens after drop weight impact test are shown in figure 6 .

Fig. 6. Deformed shapes of structures after drop weight impact test: (a) re-entrant structure, (b) arrowhead structure, (c) anti-tetra chiral structure. 


\section{Results and discussion}

\subsection{Quasi-static test results}

The load-displacement diagrams for all structures are presented in figures 7 to 9 . There is difference between the mode of collapse and the destruction of unit cells in different structures, and these differences affect the energy absorption of the structures. As shown in figures 7 to 9 , for all of the structures, there is initially a region that is a linear relationship between force and displacement. After that, in the re-entrant structure, there is a layer-wise collapse behaviour and we see a frequent rise and fall of force amounts and this trend is up to about $30 \%$ reduction in the height of the structure. Afterwards, due to the fact that a large part of the structure has been collapsed, the stiffness of the structure increases and the amount of force takes up an ascending trend. But this pattern of ascent and descent of force is not true for two other structures. In the anti-tetra chiral structure, after an initial linear region, we have an almost constant force region, and then the amount of force increases sharply that shows the densification of structure. In the case of the arrowhead structure, the first area of the graph indicates a state in which the structure resists the load and does not have much deformation. With the initiation of change in the shape of the structure, the rate of increase in the force decreases; actually, the unit cells are concentrated and collapsed in the middle of the structure, indicating the auxetic property. As the cell wall collapses and reaches together, the stiffness of the structure increases, and the amount of force becomes quite upward. The region of nearly constant force, after the peak force, indicates that the structure is uniformly deformed and the force is distributed approximately the same. The total energy absorbed by each structure under uniaxial compressive testing was calculated for all specimens.

Fig. 7. Force displacement diagram for re-entrant structure under quasi-static loading.

Fig. 8. Force displacement diagram for arrowhead structure under quasi-static loading.

In order to consider the weight of the structure, a parameter called specific energy absorption is defined, whose values are obtained by dividing the total energy absorption of the structure by its weight. The energy absorption and specific energy absorption values of the structures are shown in figures $10 \& 11$ respectively. It should be noted that to make a fair comparison condition between structures, the area under the force-displacement curve up to the 25 $\mathrm{mm}$ displacement (approximately equal to 50\% strain) is considered for energy absorption calculation. The arrowhead structure has the highest energy absorption and its energy absorption is $66 \%$ and $10 \%$ more than the reentrant and anti-tetra-chiral structures, respectively.

Fig. 9. Force displacement diagram for anti-tetra chiral structure under quasi-static loading.

Fig. 10. Energy absorption of tested structures.

Fig. 11. Specific energy absorption of tested structures.

\subsection{Low velocity impact test results}

The force-time diagrams of the models are shown in figures 12 to 14 . As shown in the figures 12 to 14 , the peak force of re-entrant structure is more than arrowhead and anti-tetra chiral structure, and the approximate time for the destruction of structures during the impact is the same. The trend of ascending and descending of the force amounts for the anti-tetra chiral and arrowhead structures is roughly the same, however in the re-entrant structure, as expressed in the analysis of the results of quasi-static loading, due to the layer-wise pattern of collapse, the number 
of ascent and descent in the force diagram are more than two other structures. It should be noted that the mechanical behaviour of the re-entrant structure in impact loading has been improved and its force amounts is in the order of two other structure.

Fig. 12 Force-time diagram of re-entrant structure derived from drop weight impact test.

Fig. 13 Force-time diagram of arrowhead structure derived from drop weight impact test.

Fig. 14 Force-time diagram of anti-tetra chiral structure derived from drop weight impact test.

\section{Conclusion}

In this study, in-plane compressive properties of three auxetic structures were studied in quasi-static and low velocity impact loading conditions experimentally. All the three models were fabricated using an advanced manufacturing technology (3D printing). Arrowhead and anti-tetra chiral structures displayed better compressive properties under quasi static loading than the re-entrant structure. But in low velocity impact loading, the performance of the re-entrant structure improved greatly and was comparable to the performance of the two other structures. Overall, it can be concluded that the anti-tetra chiral structure showed the best performance in both type of loading.

\section{References}

[1] G. Imbalzano, P. Tran, T.D. Ngo, P.V.S. Lee, Three-dimensional modelling of auxetic sandwich panels for localised impact resistance, J. Sandw. Struct. Mater. 19 (2017). doi:10.1177/1099636215618539.

[2] L.J. Gibson, M.F. Ashby, Cellular Solids: Structure and Properties, 2nd ed., Cambridge University Press, Cambridge, 1997. doi:DOI: 10.1017/CBO9781139878326.

[3] J. Vinson, The behavior of sandwich structures of isotropic and composite materials, Routledge, 2018.

[4] Y.M. Xie, Xin Ren, Raj Das, Phuong Tran, Tuan Duc Ngo, Auxetic metamaterials and structures: a review, Smart Mater. Struct. 27 (2018) 23001.

[5] M. Mir, M.N. Ali, J. Sami, U. Ansari, Review of Mechanics and Applications of Auxetic Structures, Adv. Mater. Sci. Eng. 2014 (2014) 1-18.

[6] L.J. Gibson, M.F. Ashby, G.S. Schajer, C.I. Robertson, The Mechanics of Two-Dimensional Cellular Materials, Proc. R. Soc. Lond. A. Math. Phys. Sci. 382 (1982) 25-42.

[7] R. LAKES, Foam Structures with a Negative Poisson's Ratio, Science (80-. ). 235 (1987) 1038 LP - 1040. doi:10.1126/science.235.4792.1038.

[8] K.E. Evans, The design of doubly curved sandwich panels with honeycomb cores, Compos. Struct. 17 (1991) 95-111. doi:https://doi.org/10.1016/0263-8223(91)90064-6.

[9] A. Rafsanjani, D. Pasini, Bistable auxetic mechanical metamaterials inspired by ancient geometric motifs, Extrem. Mech. Lett. 9 (2016) 291-296. doi:10.1016/j.eml.2016.09.001.

[10] C. Yang, H.D. Vora, Y. Chang, Behavior of auxetic structures under compression and impact forces, Smart Mater. Struct. 27 (2018) 25012. doi:10.1088/1361-665x/aaa3cf.

[11] A. Ingrole, A. Hao, R. Liang, Design and modeling of auxetic and hybrid honeycomb structures for in-plane property enhancement, Mater. Des. 117 (2017) 72-83. doi:10.1016/J.MATDES.2016.12.067.

[12] G. Imbalzano, S. Linforth, T.D. Ngo, P.V.S. Lee, P. Tran, Blast resistance of auxetic and honeycomb sandwich panels: Comparisons and parametric designs, Compos. Struct. 183 (2018) 242-261. doi:https://doi.org/10.1016/j.compstruct.2017.03.018.

[13] C. Qi, A. Remennikov, L.-Z. Pei, S. Yang, Z.-H. Yu, T.D. Ngo, Impact and close-in blast response of auxetic honeycomb-cored sandwich panels: Experimental tests and numerical simulations, Compos. Struct. 180 (2017) 161-178. doi:10.1016/J.COMPSTRUCT.2017.08.020. 
[14] M. Safikhani Nasim, E. Etemadi, Three dimensional modeling of warp and woof periodic auxetic cellular structure, Int. J. Mech. Sci. 136 (2018) 475-481. doi:https://doi.org/10.1016/j.ijmecsci.2018.01.002.

[15] R. Hamzehei, J. Kadkhodapour, A.P. Anaraki, S. Rezaei, S. Dariushi, A.M. Rezadoust, Octagonal auxetic metamaterials with hyperelastic properties for large compressive deformation, Int. J. Mech. Sci. 145 (2018) 96-105. doi:https://doi.org/10.1016/j.ijmecsci.2018.06.040. 


\section{Declaration of interests}

$\bigotimes$ The authors declare that they have no known competing financial interests or personal relationships that could have appeared to influence the work reported in this paper.

$\square$ The authors declare the following financial interests/personal relationships which may be considered as potential competing interests:

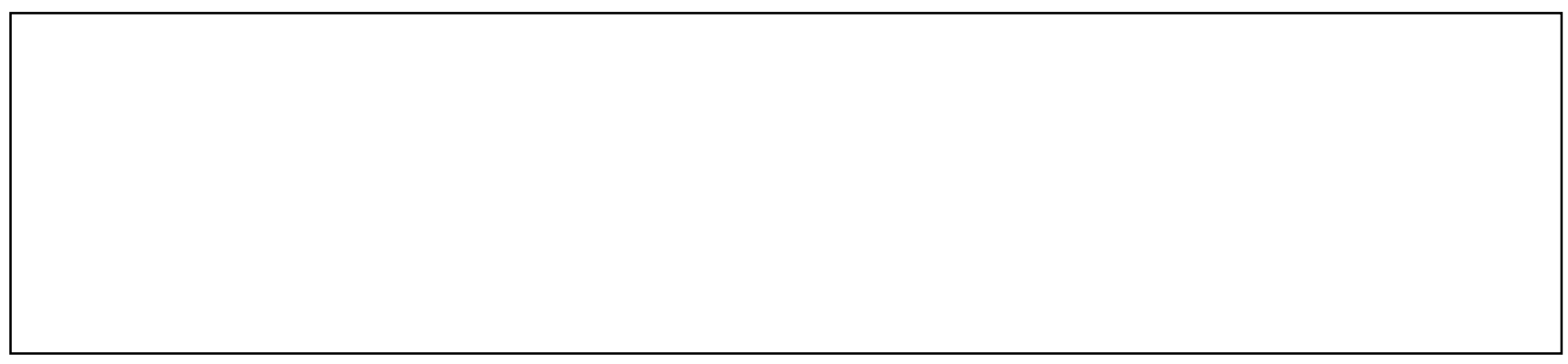




\section{CRediT author statement}

Milad Najafi: Software, Validation, Formal Analysis, Writing - Original Draft, Investigation.

Hamed Ahmadi: Conceptualization, Methodology, Writing - Review \& Editing, Visualization, Supervision, Investigation.

Gholamhossein Liaghat: Visualization, Supervision, Resources 
Click here to download high resolution image

\section{Tension}
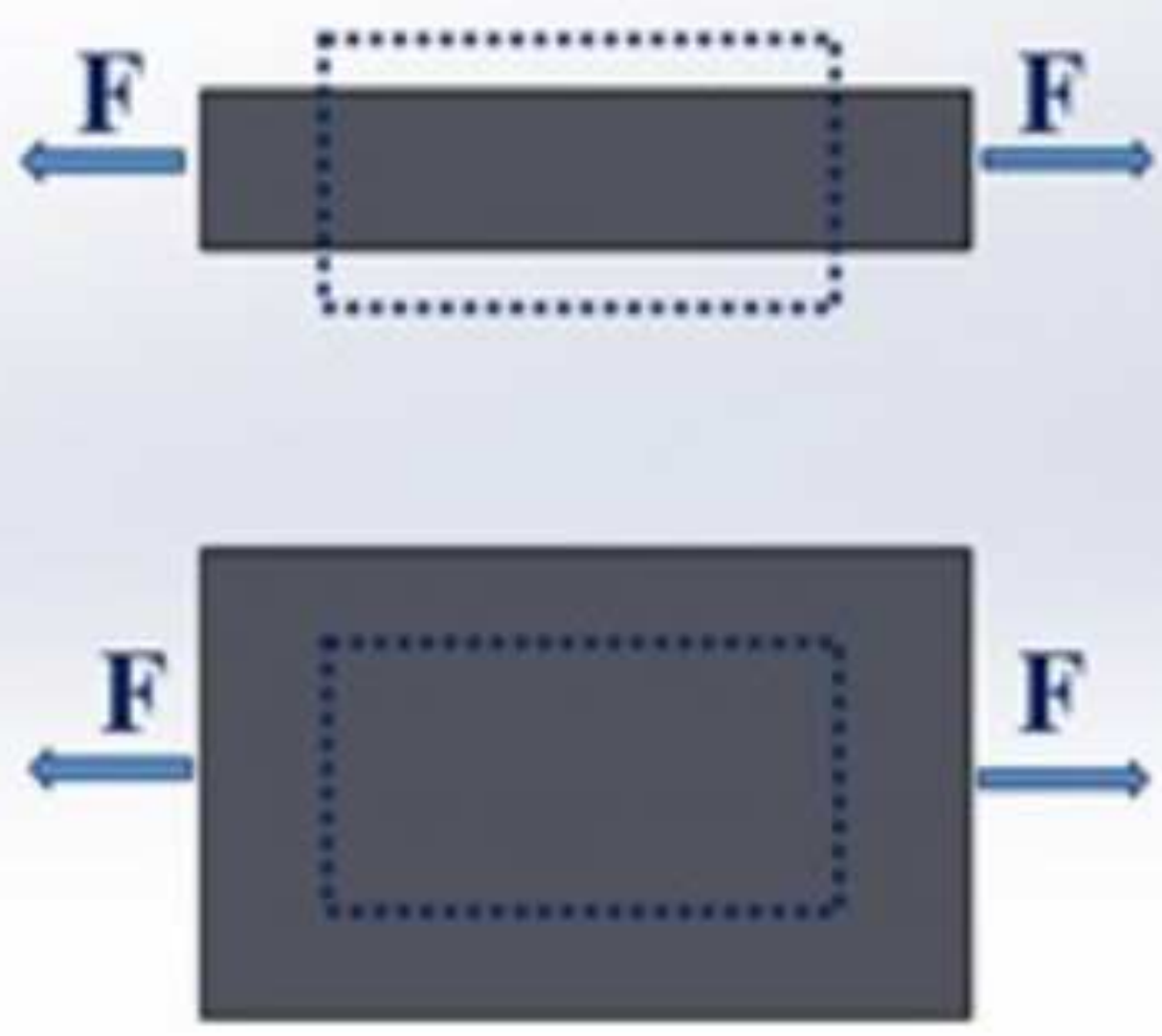

\section{Compression}

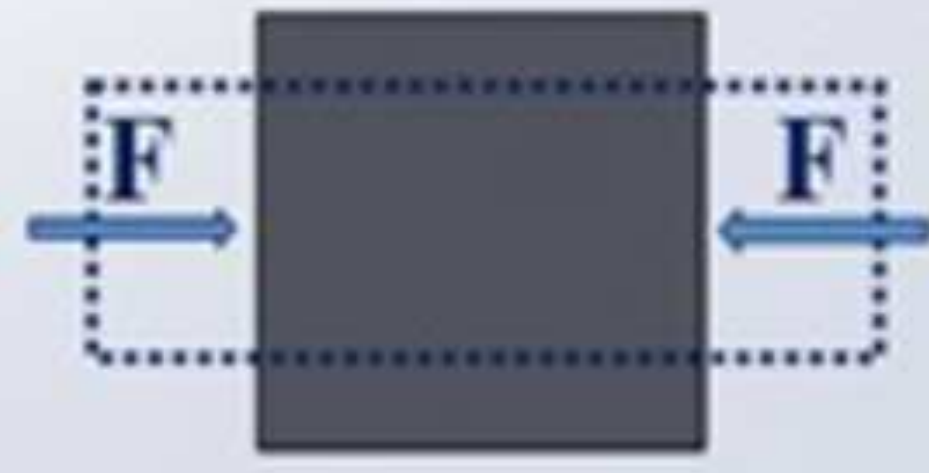

a

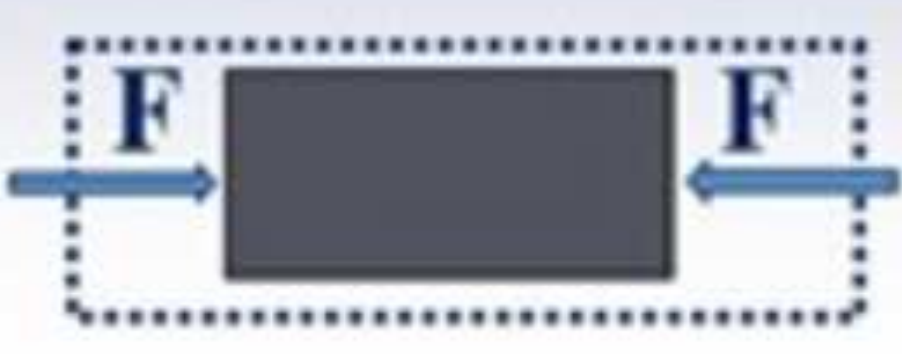

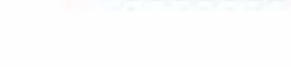

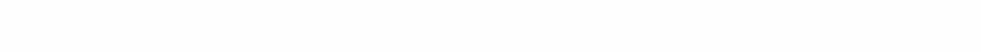




\section{Figure 2}

Click here to download high resolution image

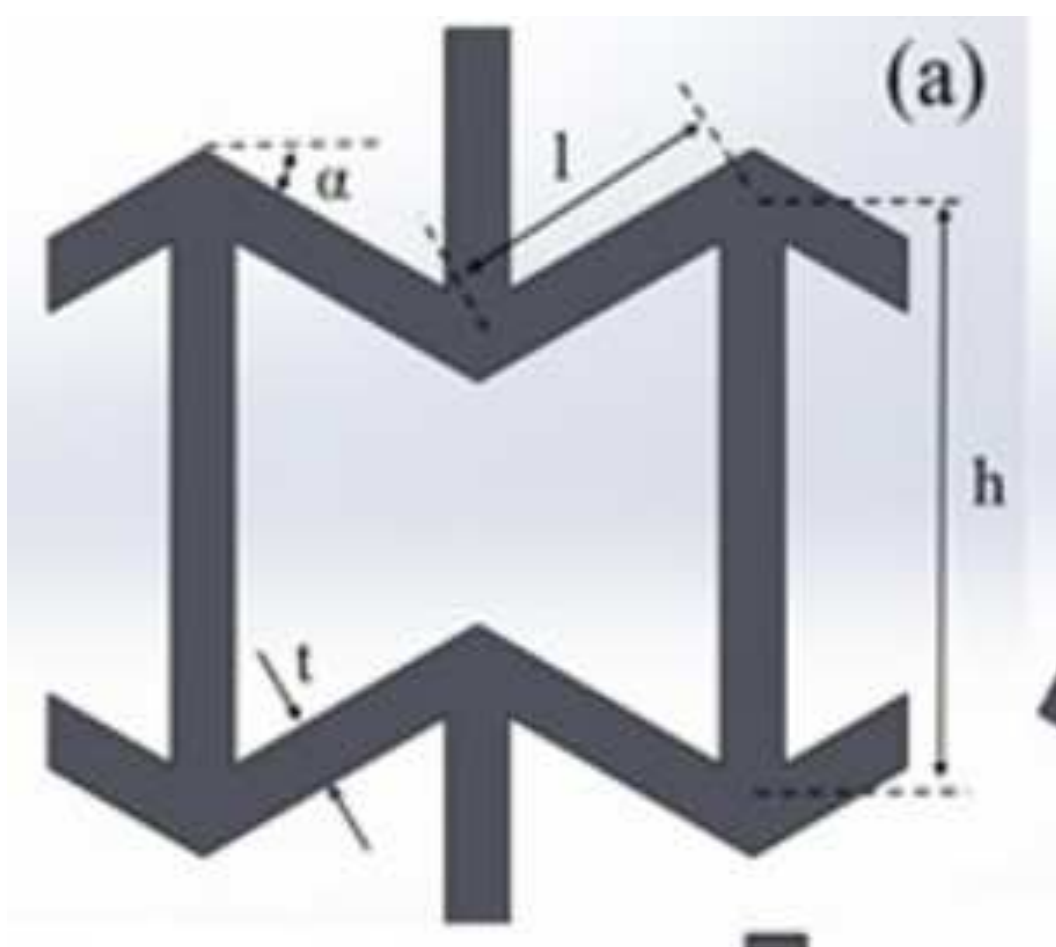

(a)
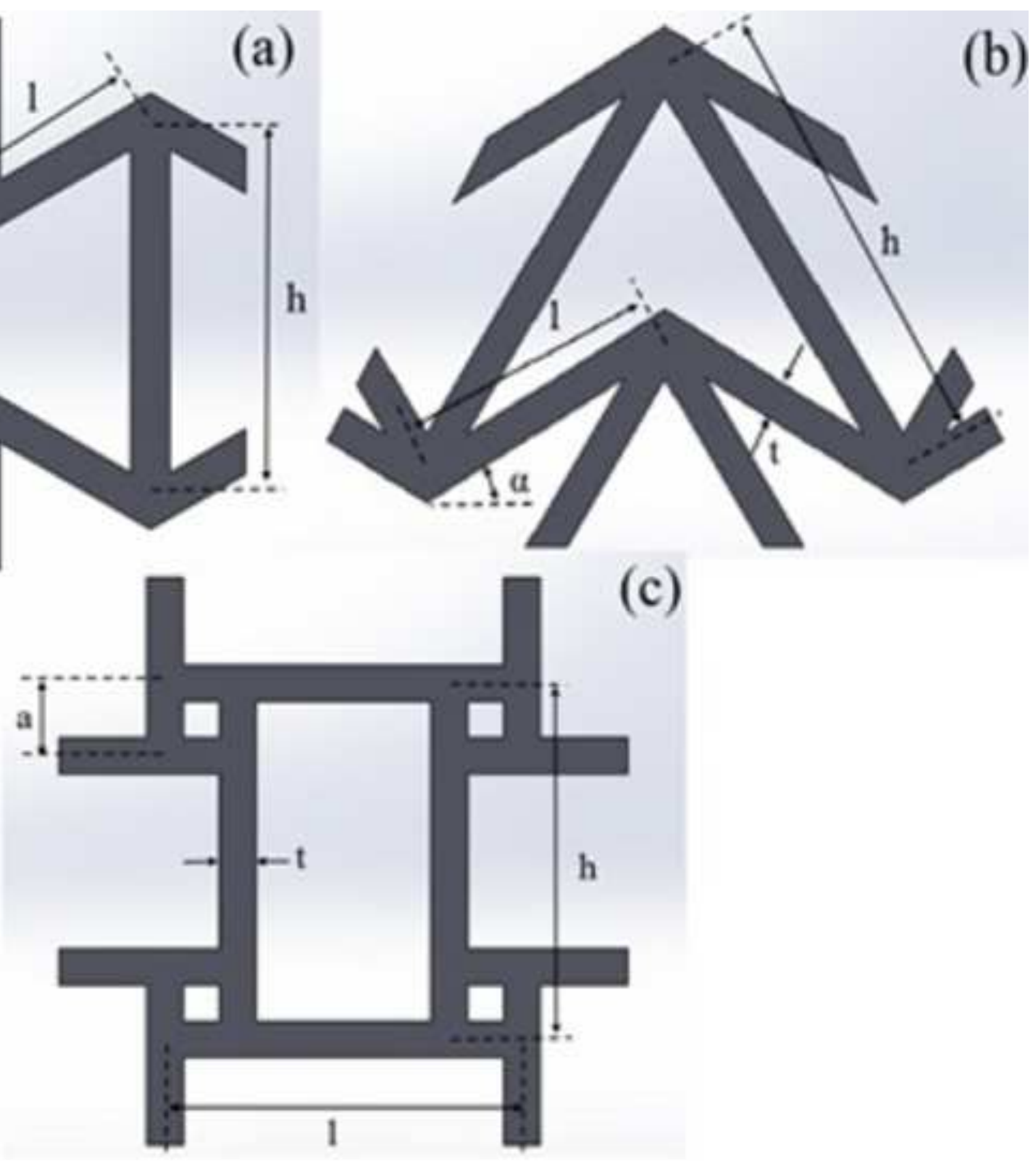

(c) 

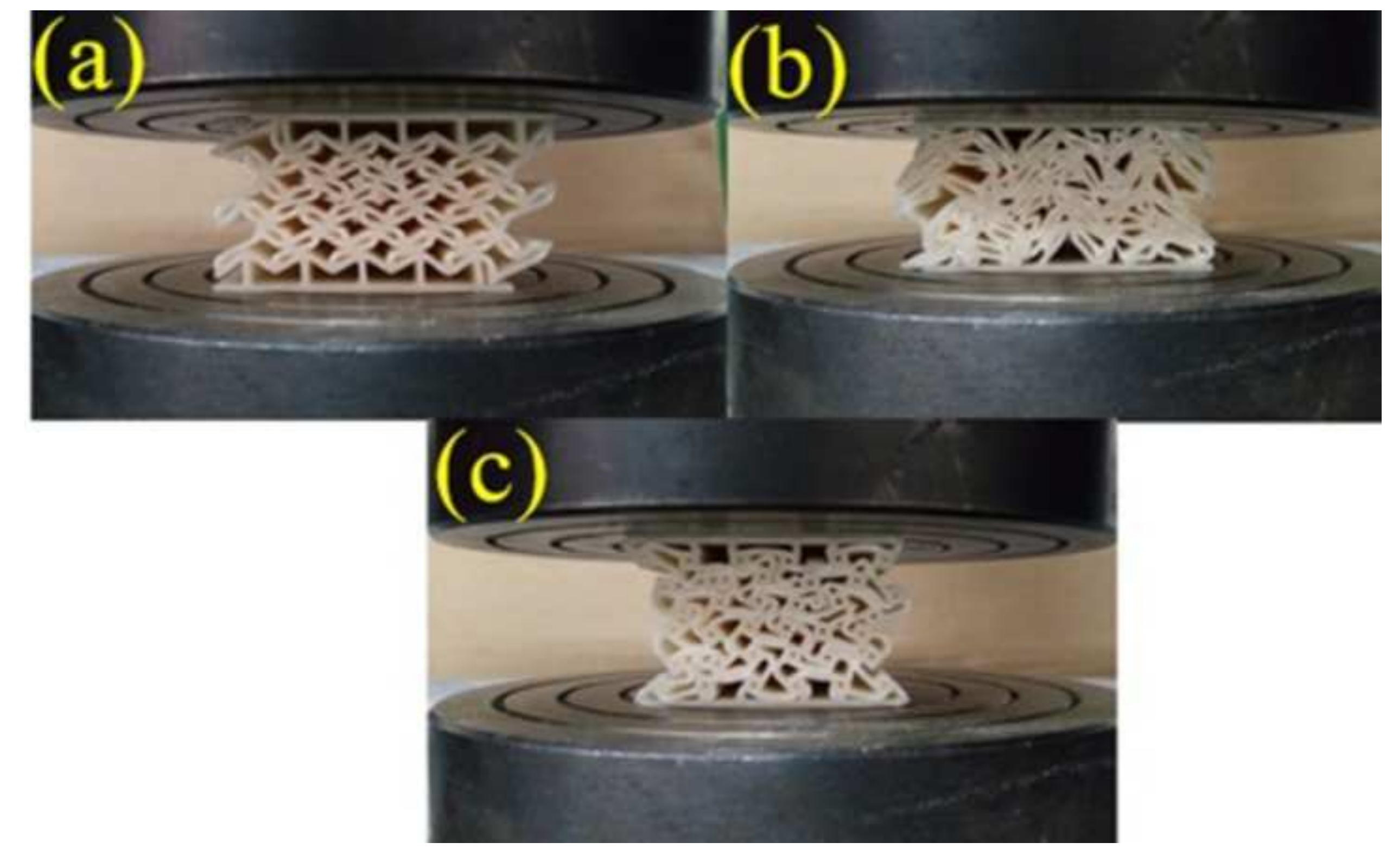


\section{Figure 5}

Click here to download high resolution image

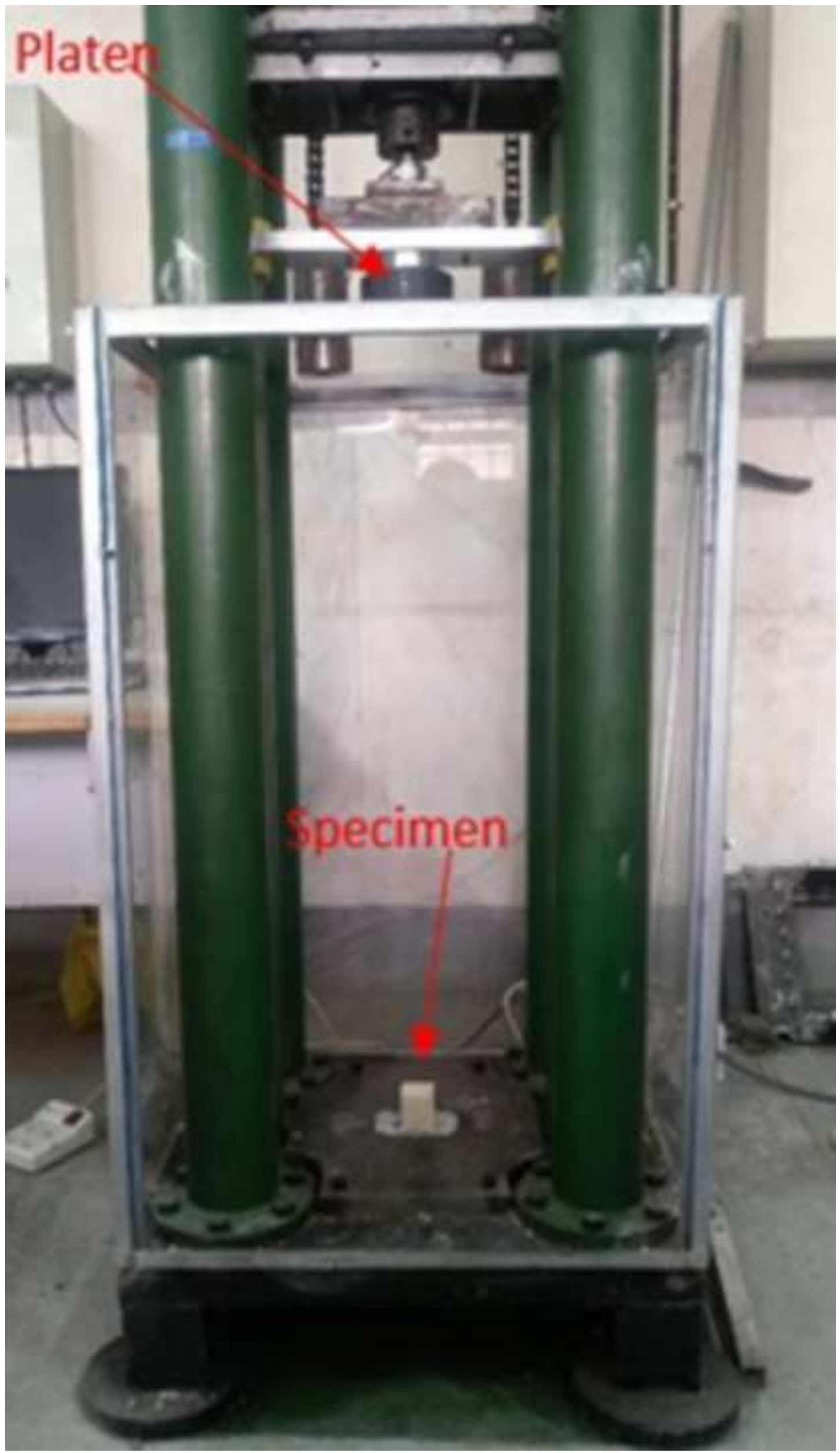


Click here to download high resolution image
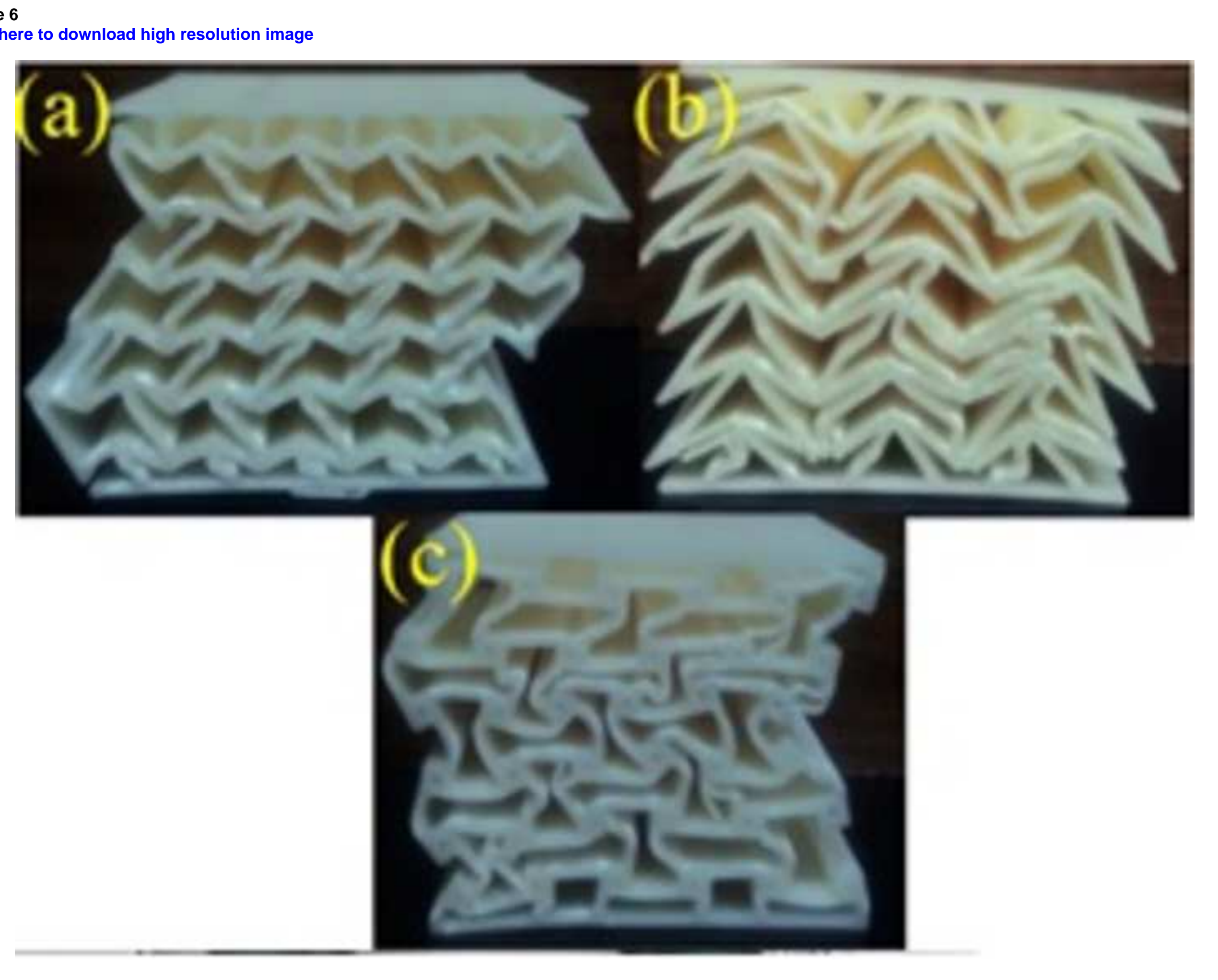

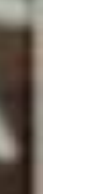

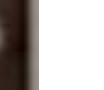
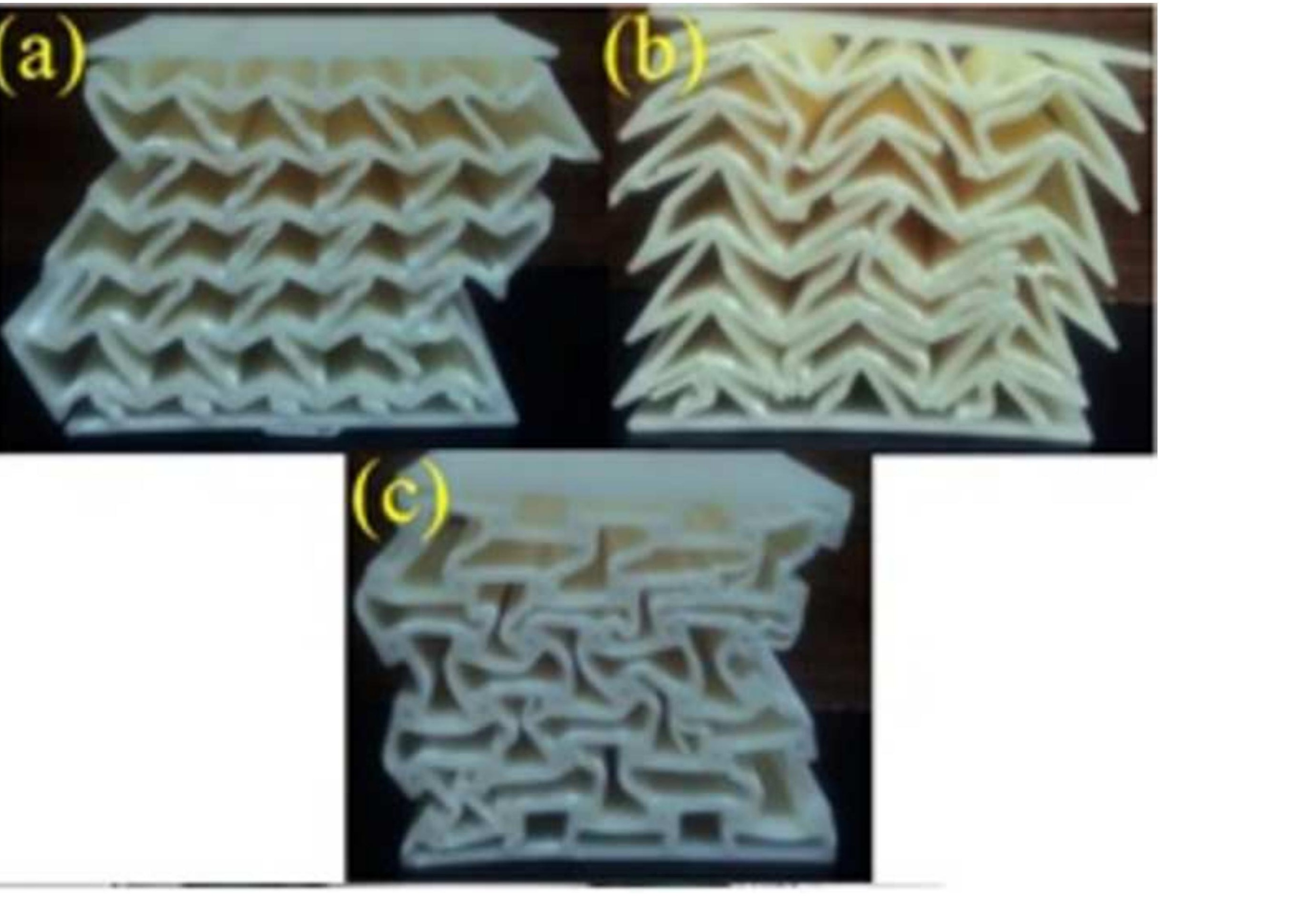
Click here to download high resolution image

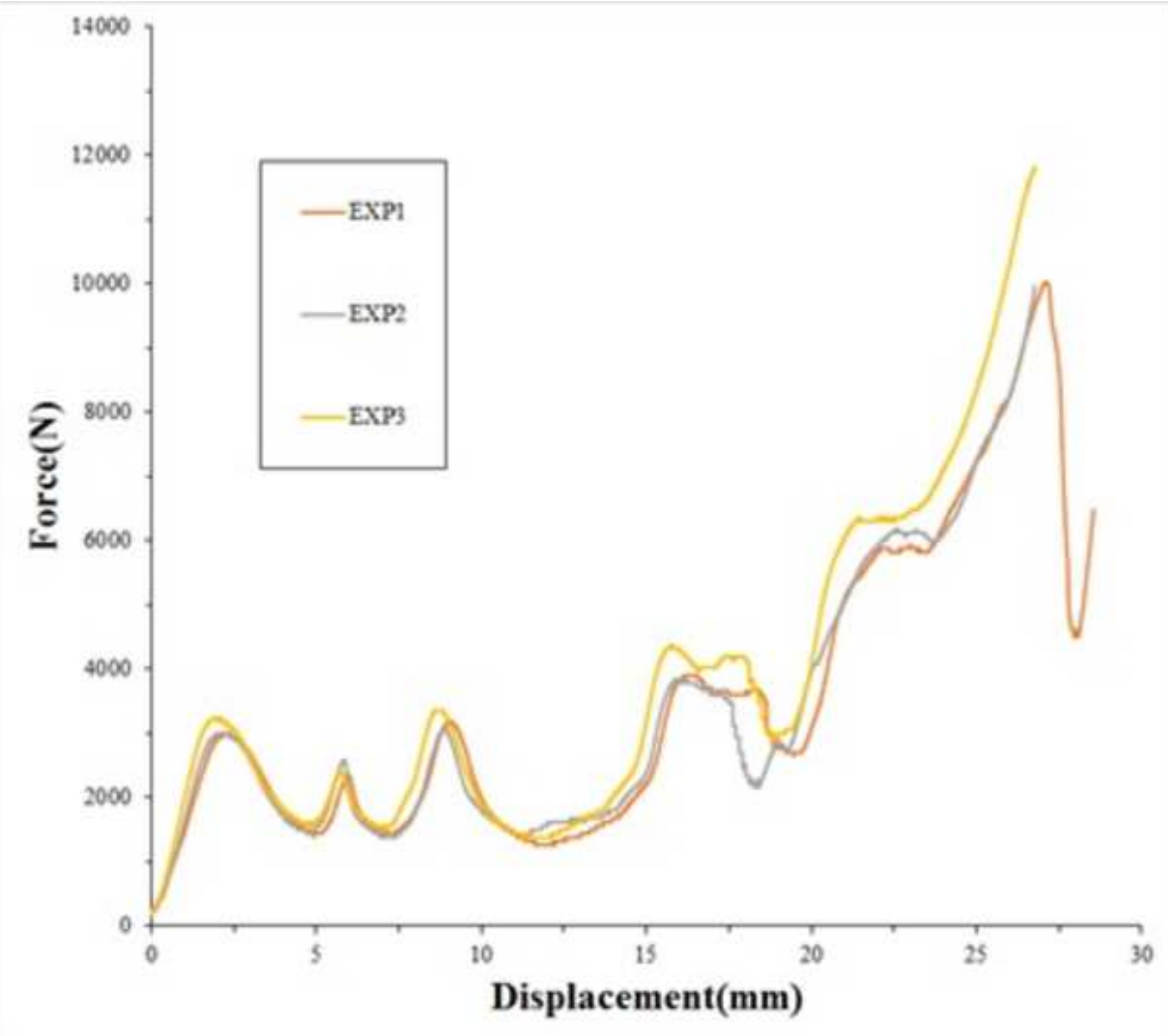


Click here to download high resolution image

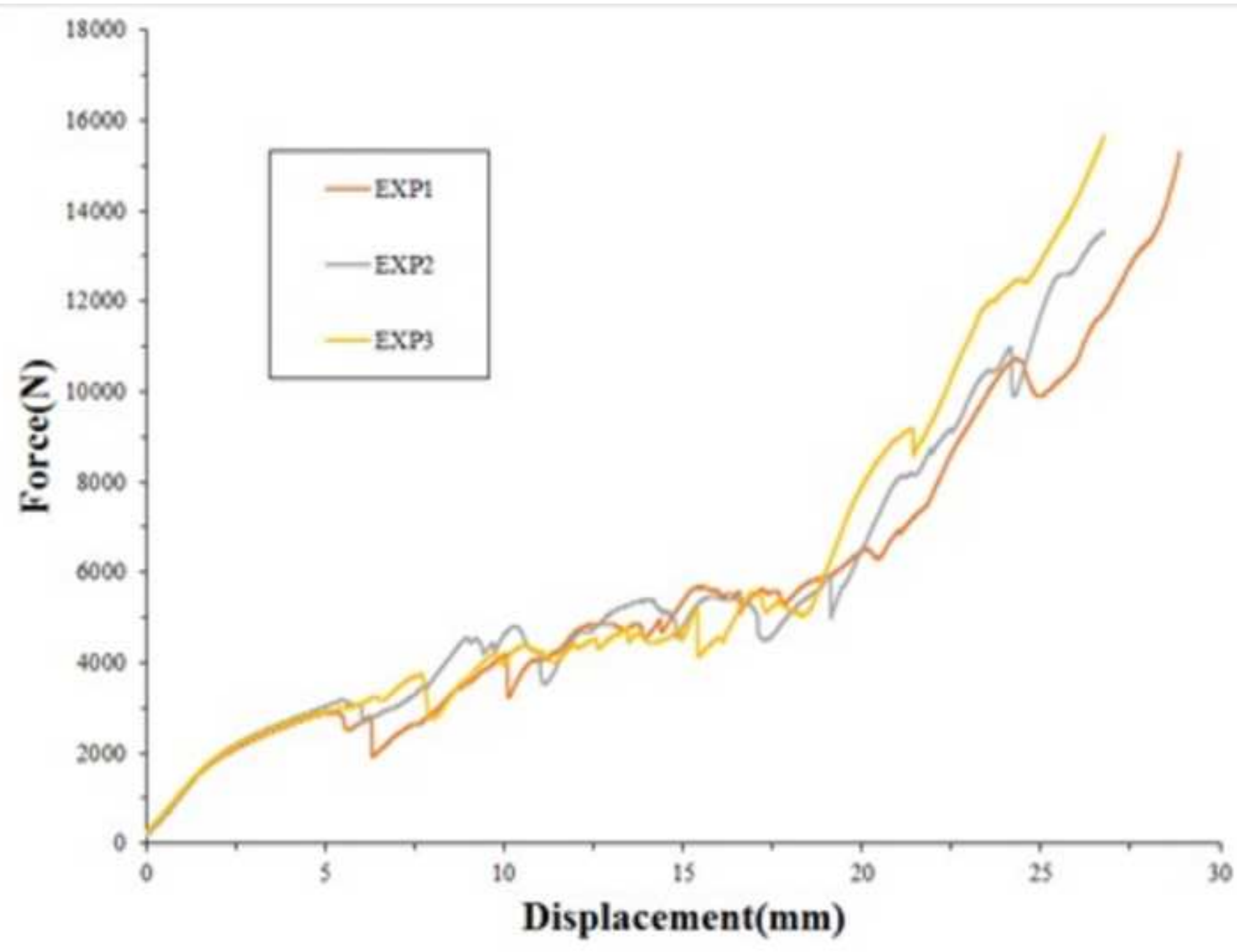


Click here to download high resolution image

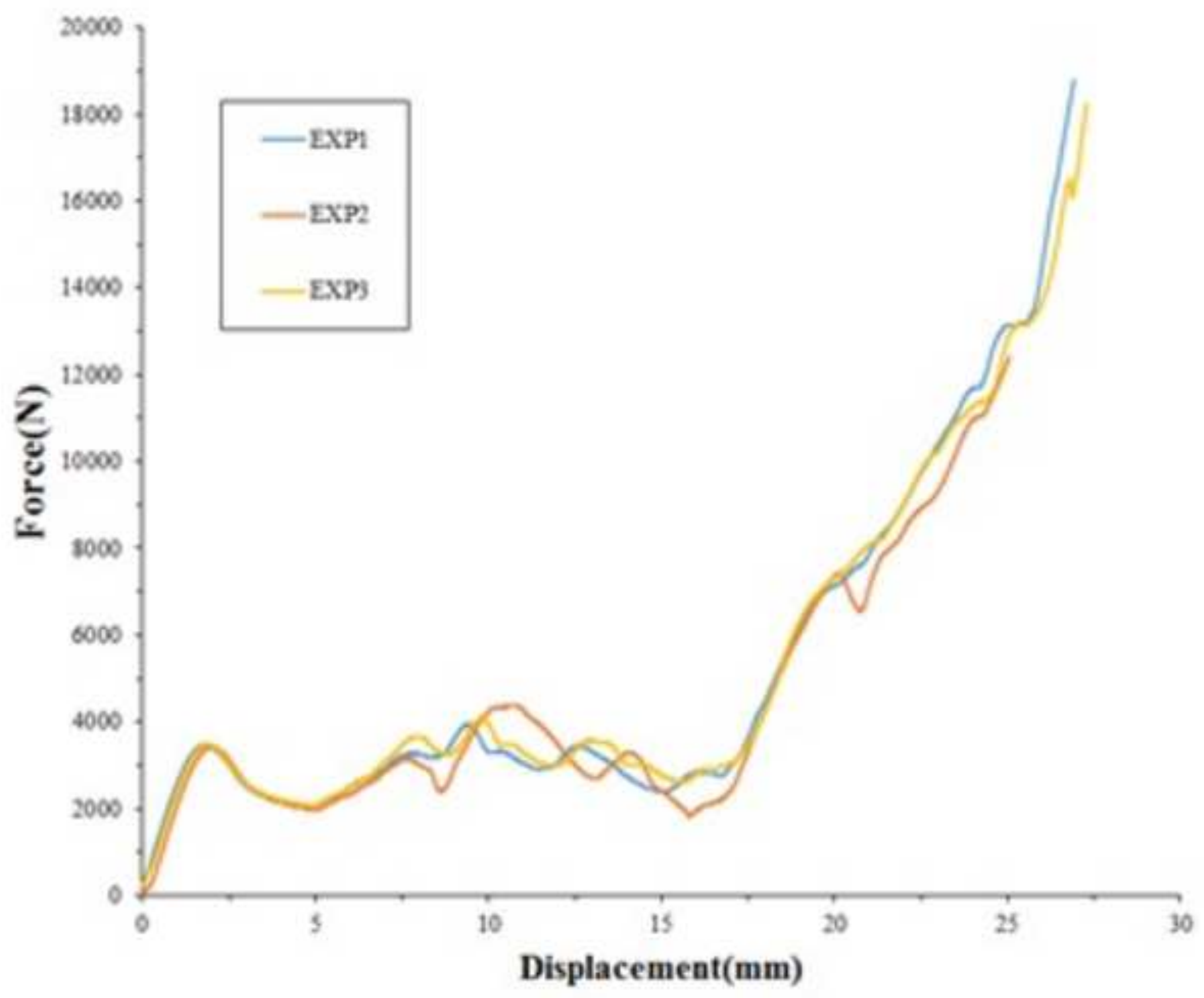




$$
11
$$


Click here to download high resolution image

4

3.5

3

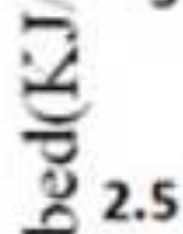

ㄴ.

80

ำ 1.5

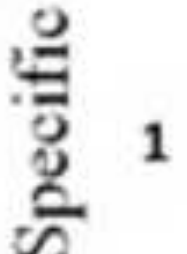

0.5

0

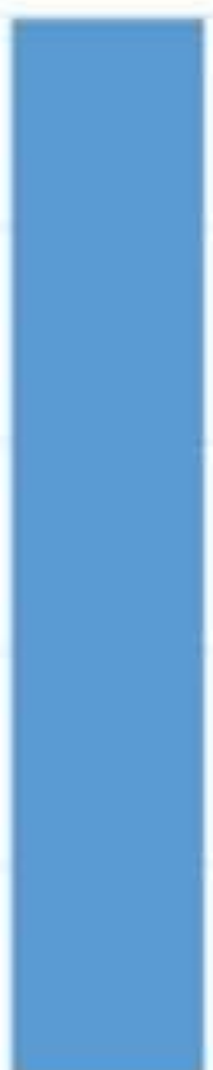

Re-entrant

Arrowhead

Anti-tetra chiral 
Click here to download high resolution image

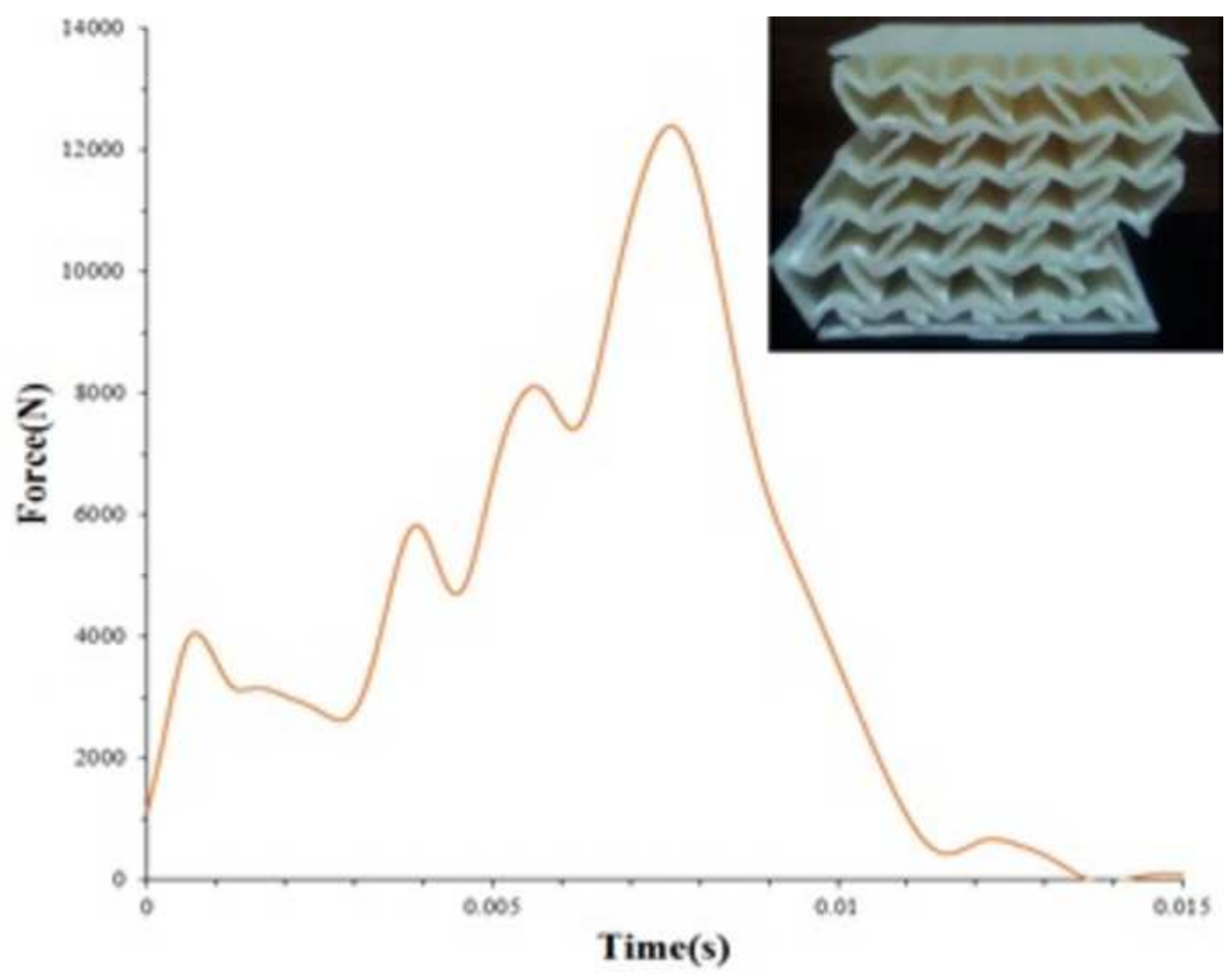


Click here to download high resolution image

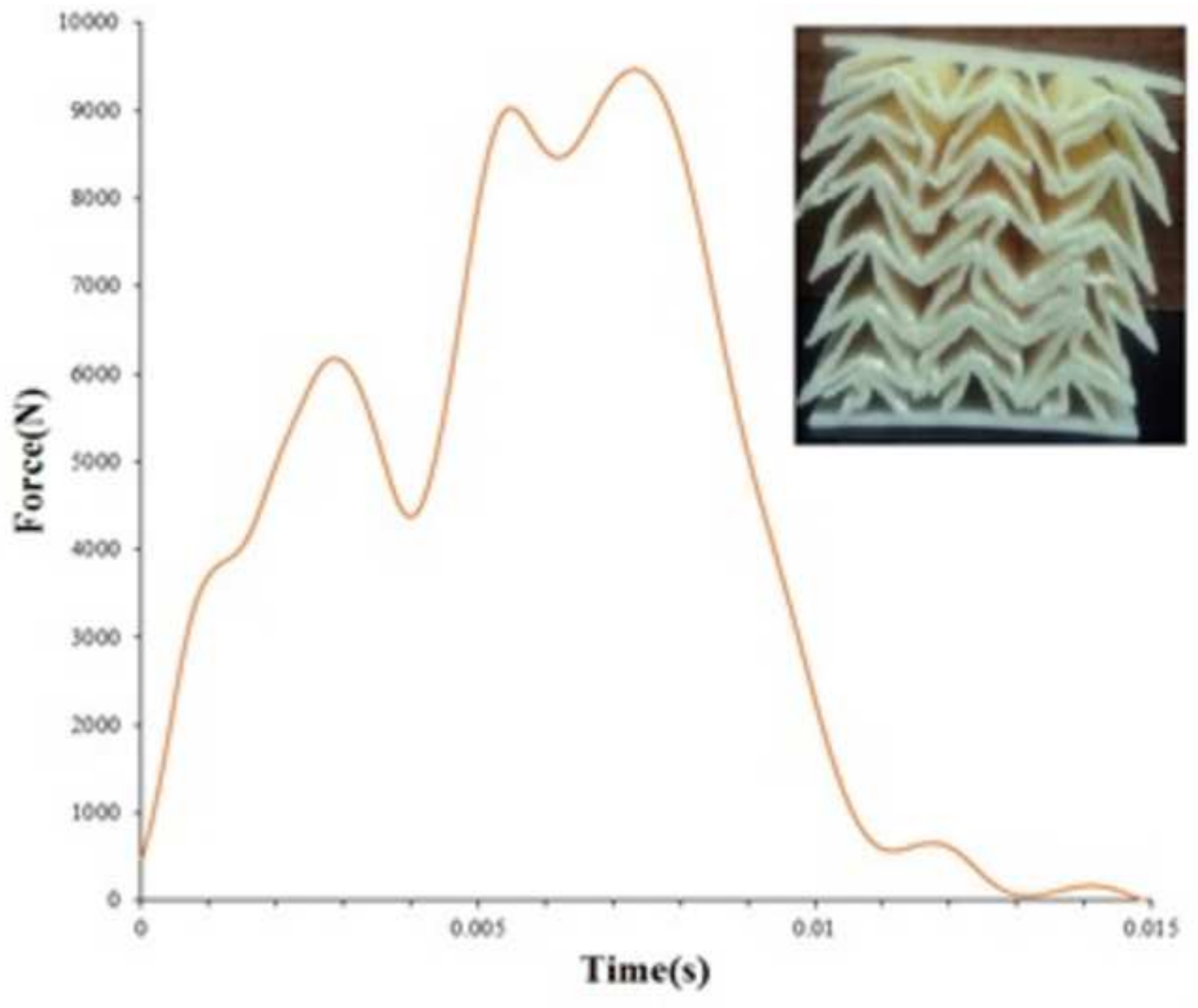


Click here to download high resolution image

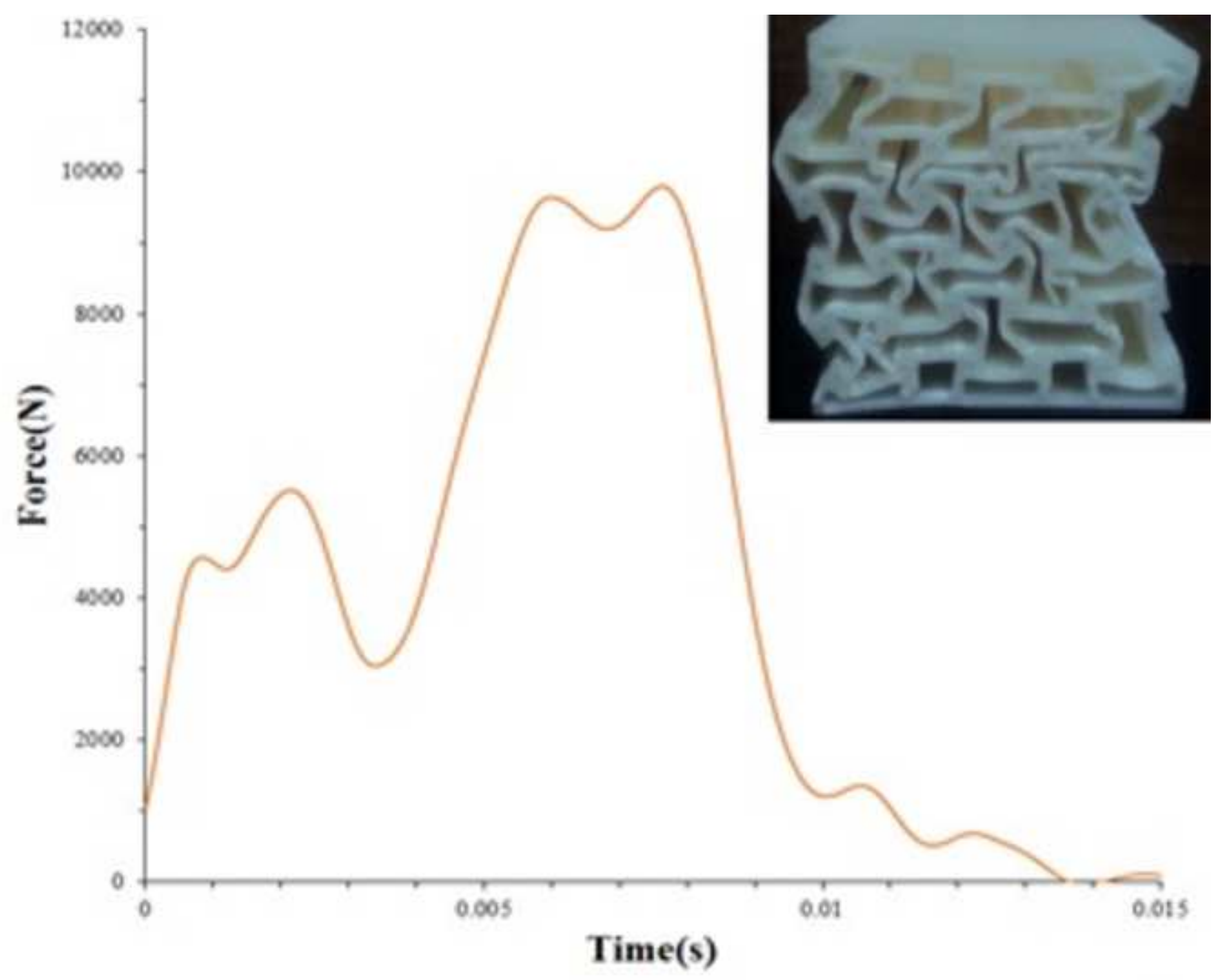

